\title{
Möglichkeiten und Aufgaben einer kritischen Sozialwissenschaft: ein Interview mit Anthony Giddens ${ }^{1}$
}

Den britischen Soziologen Anthony Giddens hier vorzustellen, dürfte nicht mehr nötig sein. Sein Name ist vertraut, und vertraut mag vielen Geograph(inn)en auch die Auswahlschwierigkeit sein, die das Volumen seiner Publikationen bereitet. Wiederholt wurde die konzeptionelle Basis sozialwissenschaftlicher Arbeit, die Giddens mit seiner «Theorie gesellschaftlicher Strukturierung» vorschlägt, sowohl von ihm selbst wie auch von englischsprachigen Geograph(inn)en in Artikeln ${ }^{2}$ und sogar in Interviewform ${ }^{3}$ zusammengefaßt.

Das Interesse an Giddens Arbeiten in der britischen und angloamerikanischen Geographie ist nicht verwunderlich, betont da doch «der führende englischsprachige Gesellschaftstheoretiker» ${ }^{4}$ die Bedeutung räumlicher Spezifität und Kontextualität und öffnet eine Türe für nunmehr wechselseitige Bezugnahmen von Humangeographie und anderen Sozialwissenschaften. Daraus folgt mehr als ein freudiges, disziplinstrategisch opportunes «Geography matters!». Beginnende Dialoge englischsprachiger Geograph(inn)en mit und um Giddens sind nicht zu überhören. Sie stützen sich zunächst auf ein «critical reading» der Strukturierungstheorie selbst, können aber auch schon auf manche Erfahrungen ihrer «critical application» (einerseits als konzeptioneller Rahmen in der geographischen Forschung ${ }^{5}$, andererseits als gemeinsame Sprache in interdisziplinärer Zusammenarbeit ${ }^{6}$ ) verweisen.

Hatte Giddens Gesellschaftskonzeption in der deutschsprachigen Geographie dagegen bisher kaum mehr als Erwähnung gefunden ${ }^{7}$, so zeichnet sich in jüngster Zeit auch hier eine kritische Auseinandersetzung mit der Strukturierungstheorie ab. Man mag darin zum Schluß kommen, Giddens großangelegtem Integrationsunternehmen sozialwissenschaftlicher Ansätze in einzelnen Bereichen nicht folgen zu wollen (ich z.B. sehe Widersprüche zwischen den impliziten Voraussetzungen mancher darin verbundenen Positionen ${ }^{8}$ ), doch verdient der von ihm vorgeschlagene Begriffsrahmen zunächst unbedingt Betrachtung. Für die gegenwärtigen Diskussionen in der Deutschschweizer Geographie, beispielsweise, erscheinen insbesondere folgende Konzepte vielversprechend:

- Eine Gesellschaftskonzeption, die «Raum» als raum-zeitliche(n) Materialität (1), - Kontext (2), Distanz (3), - begrenzte Einheit (4) berücksichtigt 9 und diese Perspektiven um einen Bezug zu Fragen von Macht und Herrschaft erweitert. (Mit seinem Zürcher Vortrag in diesem Jahr ${ }^{10}$ hat Giddens einen darauf ausgerichteten Dialog mit Schweizer Geograph[inn]en eröffnet.)

- Eine Konzeption von menschlichem Handeln, die sich weder im Voluntarismus verliert noch im Determinismus verlorengeht.

- Einen theoretischen Rahmen, der es gestattet, die bestehende Fülle räumlicher Informationen als konstituierende Elemente von Handlungen miteinander zu verbinden und in der Erklärung sozialer Abläufe fruchtbar zu machen. (Hier dürfte sich ein Anknüpfungspunkt zu B. WERLENS Überlegungen zu einer handlungsorientierten Sozialgeographie [1987] ergeben.)

- Ein Begriffssystem, das zwischen intendierten und unintendierten Handlungsfolgen und diskursivem und praktischem Wissen unterscheidet sowie mit physischgeographischen Dimensionen verbunden werden kann, und damit für humanökologische Fragestellungen geeignet erscheint. (Der Beitrag von C. JÄGER und D. STEINER in diesem Heft, S. 133, weist in diese Richtung.)

- Ein Modell von "Gesellschaft» als rekursiver Pro$z e \beta^{11}$, das als Hintergrundkonzeption die Formen und Voraussetzungen der gesellschaftlichen Verwirklichung von (z.B. entwicklungsstrategischen oder humanökologischen) Anliegen der Geographie hervortreten läßt. Es gestattet, die gesellschaftskritischen Beiträge des Faches selbst, reflektiv in die Betrachtung einzubeziehen.

Diesen letztgenannten Bezugspunkt möchte ich mit dem folgenden Interview mit A. Giddens in allgemeinerer Form aufgreifen: Anknüpfend an die Diskussionen um die «Rolle der Geographie in der Gesellschaft» beim Berner und Fribourger Geographentag 12 stelle ich in der abstrakten Sprache einer Gesellschafts- und Wissenschaftstheorie die Frage, ob die Humangeographie leisten kann, was ein Sprecher dort gefordert hat ${ }^{13}$ : In angewandter Arbeit müßten Geograph(inn)en erklärende Problemanalysen, kritische Stellungnahmen und wohlbegründete Lösungsvorschläge erbringen können.

Dagmar Reichert, Geographisches Institut der Universität Wien, Universitätsstr. 7, A-1010 Wien 
Geographische Forschung müsse allgemeine Richtlinien und eine Wissensgrundlage in Form von Theorien (u.a. zur Prognose) dafür bereitstellen. - Damit ist auch eine weitere Frage verknüpft: Auf welcher Basis kann sich die Geographie kritisch mit gesellschaftlichen Normen und Werten auseinandersetzen, um, wie M. BOESCH (1986, S.150) treffend sagt, nicht länger ihre politische Unschuld um den Preis ihrer Belanglosigkeit zu bewahren?

Hier, wie im folgenden Interview, geht es darum, die Möglichkeiten und Aufgaben einer Humangeographie als engagierter Sozialwissenschaft von innen, aus der Selbstreflexion dieser Wissenschaft heraus, zu klären. Zwischen der verbreiteten Skepsis gegen wissenschaftliche Untersuchungen auf der einen Seite und pragmatisch-technokratischer Wissenschaftsgläubigkeit auf der anderen; zwischen relativistischer Resignation im Selbstverständnis der Forschenden, und absolutistischer Expertenmanier im Dienste potenter Auftraggeber, gilt es immer wieder, nach einem verantwortbaren Arbeitsbereich für eine kritische Humangeographie zu fragen.

D.R.: Welche Rolle kommt Ihrer Meinung nach den Sozialwissenschaftlern in der Gesellschaft zu? Sehen Sie eine Möglichkeit für eine kritische Sozialwissenschaft?

A.G.: Man muß das vor dem Hintergrund des Verhältnisses zwischen Sozialwissenschaften und Moderne sehen. Die modernen Sozialwissenschaften sind nicht allein aus dem Versuch entstanden, gesellschaftliche Veränderungen zu verstehen. Von Anfang an waren sie eng damit verbunden, unsere $\mathrm{Ge}$ sellschaften zu konstituieren. Darin sind sie wahrscheinlich sogar weit wesentlicher, als die meisten Sozialwissenschaftler behaupten würden. Für mich hat ihre gesellschaftliche Rolle drei Aspekte: Der erste ist reine Informationsbereitstellung. Er entspringt aus der Tatsache, daß moderne Gesellschaften von regelmäßiger Information über Scheidungsraten, Inflation oder was auch immer abhängen.

Den zweiten Aspekt kann man als die reflexive oder zirkuläre Beziehung zwischen Sozialwissenschaftlern und ihrem Forschungs «gegenstand» beschreiben. Im Gegensatz zu naturwissenschaftlichem Wissen ist sozialwissenschaftliche Information nicht passiv. Sie wird von vielen Leuten in verschiedener Weise aufgenommen, um die sozialen Verhältnisse zu beeinflussen, die darin beschrieben werden. Verschiedene Formen von Verantwortung gehen daraus hervor, denn wer diese Informationen liefert, darf nicht glauben, sie/er könnte sich von aller Verantwortung für die Verwendung dieses Wissens gegen andere freisprechen. Dies gilt, obwohl man nicht erwarten kann, daß jede(r) Sozialwissenschaftler(in) vollständig kontrollieren kann, was mit seinen oder ihren Ideen geschieht. Sie entwischen ganz zwangs- läufig ihren Schöpfern - aber diese Reflexivität selbst zu studieren, das sollte Teil unserer Verantwortlichkeit sein. Der dritte Aspekt der gesellschaftlichen Rolle der Sozialwissenschaftler besteht in der Kritik an sozialen Institutionen oder allgemeinen Werten. Ideologiekritik ist eine ihrer wesentlichsten Aufgaben, und zweifellos die schwierigste. Ich verstehe «Ideologie» nicht einfach als falsches Bewußtsein, und auch nicht als die gegenteilige Auffassung, für die alle Arten von Ideen, die das Bewußtsein beeinflussen, als Ideologien gelten, sondern eher als Ideen, die mit Herrschaft verbunden sind ${ }^{14}$. Deshalb ist es für mich eine der grundlegenden Aufgaben, die Formen zu kritisieren, in denen bestimmte Bedeutungssetzungen mit Machtsystemen verbunden werden. Dies setzt nicht von vornherein voraus, da $\beta$ die Ideen, die dabei angewendet werden, wahr oder falsch sind. Diese Frage muß man einklammern, wenn man Ideologiefragen diskutiert.

D.R.: Wenn man sie einklammern muß - woher haben Sozialwissenschaftler dann gegenüber jenen, die sie kritisieren, eine privilegierte Erkenntnisposition, d.h. einen legitimen Stand für solche Ideologiekritik?

A.G.: Nur daraus, daß sie mit anderen Sozialwissenschaftlern in jenem kritischen Dialog stehen, der grundsätzlich eine systematischere Einschätzung der Beziehung zwischen bestimmten Informationen und Machtsystemen gestattet, als sie anderen Menschen im Alltag möglich ist. Ich glaube nicht, daß Sozialwissenschaftler über eine epistemologisch privilegierte Position verfügen oder daß diese Frage viel mit den traditionellen Problemen einer Wissenssoziologie oder mit der Zirkularität des Wissens zu tun hat. Machtstrukturen in der Gesellschaft entdecken zu können, ihren Zusammenhang mit Formen der Bedeutungssetzung aufzuzeigen, mit Ideensystemen, welche jedoch auch die Alltagspraxis leiten, dies hat nicht notwendigerweise etwas mit der Frage nach der Position sozialwissenschaftlicher Beobachter relativ zu anderen Beobachtern zu tun. Mannheim hat da mit seiner Kritik an Ideologie eine falsche Richtung vorgeschlagen ${ }^{15}$. Zirkularität ist ein Problem für Erkenntnis in den Sozialwissenschaften, aber keines, das spezifisch mit der Frage der Ideologiekritik verbunden wäre.

D.R.: Aber ist Ideologiekritik nicht nur eine Form sozialwissenschaftlicher Aussagen und aus den selben Gründen kontextabhängig und relativ?

A.G.: Ideologiekritik und epistemologische Fragen sollten getrennt werden. Es ist ein Irrtum, zu glauben, Ideologiekritik setze eine Betrachtung der Zirkularität von Wissen, seiner eigenen Verknüpfung mit Ideologie voraus - zumindestens Ideologiekritik, wie ich sie verstehe. 
Die Frage nach der Art sozialwissenschaftlichen Wissens, nach seiner Begründung, oder die Frage, ob Werte objektiv kritisierbar sind - das, womit Habermas sich beschäftigt - das besteht daneben, getrennt davon. Es ist nicht zu erwarten, da $B$ man eine sichere epistemologische Basis finden wird, von der aus Werte kritisierbar sind. Wahrscheinlich muß man es versuchen, aber ich kann mir nicht vorstellen, daß man eine bestimmte Lösung finden wird, in der alle Beteiligten einer Art von Ideologiekritik zustimmen würden. Habermas' Vorschlag wird kaum eine Minorität der Sozialwissenschaftler überzeugen, geschweige denn die meisten anderen Menschen $^{16}$. Auf der anderen Seite halte ich auch nichts von einer historischen Position, die Werte einzig in geschichtlichen Zusammenhängen analysieren will. Ich vertrete hier eher eine Position, die, sagen wir, etwa in der Richtung von Adorno liegt. Ich kann mir nicht vorstellen, wie man eine Kritik von Werten jemals in einem logischen Rahmen oder in empirischen Entdeckungen begründen kann.

Auf der anderen Seite glaube ich, daß die ganze Frage der Kritik von Werten übertrieben werden kann, jedenfalls wenn man die praktische Arbeit und die praktischen Konsequenzen der Sozialwissenschaften - oder eben auch Ideologiekritik - betrachtet. Es gibt eine ganze Menge allgemeiner Werte, die sicherlich genügend akzeptiert sind, um bestimmte Gegebenheiten auf der Basis ihrer Maßstäbe zu kritisieren. Dies gilt z.B. für die Sicherung eines angemessenen Lebensstandards für die Mehrheit der Bevölkerung, für die Gewährung demokratischer Mitbestimmung, für den Schutz des Ökosystems der Erde, die Förderung von Gleichheit zwischen Frauen und Männern oder zwischen verschiedenen Rassen ...

D.R.: Genügen diese allgemeinen Werte in den Fällen, in denen solche Fragen in gesellschaftlichen Konflikten akut werden - ich denke z.B. an sozialwissenschaftliche Gutachten über die Versorgung mit Sozialleistungen oder über Kosten und Nutzen von Kernkraftwerken -, genügen sie, um sozialwissenschaftliche Stellungnahmen (und die politische Funktion sozialwissenschaftlicher Stellungnahmen) zu rechtfertigen?

A.G.: Ich glaube nicht, daß es sich in solchen Fällen um Werte dreht, eher um die Frage nach Prioritäten, Zweckgerichtetheit und eine Einschätzung der Konsequenzen. Im Falle der Diskussion um Atomkraftwerke würde niemand behaupten, sie sollten gebaut werden, auch wenn sie gefährlich wären, d.h. mit großer Wahrscheinlichkeit Millionen Leute umbringen würden. Da geht es darum, ob sie wirklich gefährlich sind, und nicht darum, was man unter «Gefährlichkeit» versteht. Befürworter und Gegner haben den selben Begriff von Gefährlichkeit. Das ist ein gutes Beispiel für das, was ich sagen will, kein Gegenbeispiel. Es gilt für die meisten umstrittenen Themen des sozialen Lebens, erst wenn man mit Fällen wie Auschwitz konfrontiert wird, wird es schwierig. -

Aber ich vertrete keinen Werterelativismus, sondern glaube, daß eine bestimmte, beschränkte $\mathrm{Be}$ urteilung von Werten möglich ist. - Nicht aber eine umfassende logische Verteidigung oder eine transzendentale Begründung eines Wertesystems. Eine schlüssige epistemologische Begründung scheint, soweit ich sehe, nur in einem theokratischen System möglich. Es gibt diese Art von Zirkularität im Innersten der Rationalität. Habermas und andere wollen sie durchbrechen, aber es scheint, daß man mit ihr leben muß, mit der Frage: Wie kann man Vernunft, oder Verpflichtung zu Vernunft, vernünftig begründen?

D.R.: Welche Bedeutung hat Zirkularität und Selbstreferenz in den Sozialwissenschaften? Geben Sie ihr insofern Bedeutung, als sie die Begründung von Wissen zum Problem macht oder als sie Verständigung ermöglicht, oder darin, daß sie den grundlegenden Unterschied zu den Naturwissenschaften darstellt...?

A.G.: Das hängt davon ab, was mit Zirkularität und Selbstreferenz gemeint ist. Beispielsweise, daß zwischen der Tätigkeit der Sozialwissenschaftler und ihrem Forschungsbereich eine ständige, wechselseitig modifizierende Verbindung besteht, daß sozialwissenschaftliche Konzepte an der Gesellschaft geprüft werden, aber auf der anderen Seite auch - was bisherige Beschreibungen zu wenig betont haben selbst wieder ihren «Gegenstand» beeinflussen. Oder die Zirkularität darin, daß man, wie Winch gezeigt hat, eine Gesellschaft nicht beschreiben kann, wenn man noch nicht weiß, was ihre Mitglieder wissen - in welche Metasprache man das später auch übersetzen mag. Das enthält vielleicht jenes Element von Selbstreferenz, das Sie meinen. Um ein Schachspiel verstehen zu können, muß man das wissen, was die Spieler wissen, den Unterschied zwischen König und Turm, Spielstrategien, . . .

Der Begriff der doppelten Hermeneutik, die Bezeichnung der Tatsache, daß man es in den Sozialwissenschaften mit zwei Bedeutungsebenen zu tun hat, da $B$ Sozialwissenschaftler mit einem geordneten Begriffssystem etwas beschreiben, das selbst schon in einem Begriffssystem geordnet ist, ... hat mit dieser Zirkularität insofern zu tun, als er besagt, daß dieses wechselseitige Verhältnis von Verständnis und Vorverständnis in den Sozialwissenschaften auf zwei Ebenen besteht.

D.R.: Sie haben zuvor von der gesellschaftlichen Verantwortung einer kritischen Sozialwissenschaft gesprochen. Mit welcher Art von Information, der Suche nach welcher Art von Wissen, könnte dieser Verantwortung, Ihrer Meinung nach, entsprochen 
werden? Wären hier Erklärungen und Vorhersagen auf der Basis allgemeiner Regelmäßigkeiten notwendig (und wenn, Regelmäßigkeiten im Bereich von empirischen Phänomenen, von verursachenden Prozessen oder der abstrakten Logik einer Entwicklung), und/oder bräuchte es die Darlegung von Alternativen für bestehende Sichtweisen, Ziele und Strategien (auf der Basis der Beschreibung spezifischer räumlich, zeitlich, kulturell unterschiedlicher Lebensformen und der kreativen Findung neuer Möglichkeiten) und/oder . . . ?

A.G.: Das möchte ich mit einem Verweis auf zwei Arten von Regelmäßigkeiten oder Generalisierungen beantworten, die Sozialwissenschaften suchen. Einerseits gibt es Generalisierungen, die auf der Kenntnis der Konventionen beruhen. Solche Regelmäßigkeiten unterscheiden sich wesentlich von jenen der Naturwissenschaften, denn sie werden von Handelnden in Anbetracht eines Wissens über ihr Tun hervorgebracht. Winch erläutert das am Beispiel vom Anhalten bei Verkehrsampeln. Was wie eine naturgesetzliche Regelmäßigkeit aussehen mag, kommt zustande, weil Fahrer die Konventionen des Verhaltens im Straßenverkehr kennen und die Gründe akzeptieren, warum sie ihnen folgen sollten. Die an'dere Art der Generalisierungen ist nicht so verschieden von der traditionellen, naturalistischen Auffassung. Sie beruhen im wesentlichen auf unintendierten Konsequenzen und führen über Feedbackmechanismen zu Regelmäßigkeiten, die nicht aus konventionsgeleitetem Handeln entstehen. Ein Beispiel dafür - ich habe es in verschiedenen Texten erwähnt - ist die Spirale der Verarmung, ein Feedbackproze $\beta$, der von niemandem intendiert wird, obwohl er von intentionalem Handeln abhängt. Aber was auf den ersten Blick wie eine naturwissenschaftliche Generalisierung aussieht, unterscheidet sich doch grundsätzlich von ihr, denn Regelmäßigkeiten vom zweiten Typ können mit Hilfe der Regelmäßigkeiten des ersten Typs verändert werden. Genau darauf beruht die Möglichkeit einer kritischen Sozialwissenschaft zu veränderndem Einzugreifen, denn Wissen über soziale Prozesse bestimmt das Handeln der Menschen und damit die Art der Regelmäßigkeiten, die sich daraus ergibt. Ist also z.B. Wissen über die Spirale der Armut gewonnen, so besteht die Möglichkeit, unintendierte Konsequenzen in konventionsgeleitetes und bewußtes soziales Handeln überzuführen und die Bedingungen zu ändern, die zur Reproduktion der Spirale der Armut beitragen.

D.R.: In der Geographie hat es in letzter Zeit erneute Diskussionen um den Nutzen von Generalisierung, von sehr allgemeinen Beschreibungen und Erklärungen gegeben ${ }^{17}$. Dabei wurde auch Ihre Strukturierungstheorie als Beispiel für jene «grand theories» kritisiert ...
A.G.: Im Falle der Strukturierungstheorie, oder von Gesellschaftstheorien allgemein, muß es nach meiner Meinung "grand theory" geben. Man muß versuchen festzulegen, was man unter Handelnden versteht, wie man sich soziale Institutionen vorstellt, wie soziale Organisation und Kultur in Raum und Zeit strukturiert sind - das sind auch «grand concerns». Mit diesen allgemeinen Konzepten kann man dann jene beschränkten Generalisierungen vornehmen, die über historische Perioden möglich sind, und die sind sehr beschränkt. Man kann z.B. «Geschichte» nicht beschreiben, ohne zu bemerken, daß sich das, was darunter verstanden wird, je nach dem Bewußtsein der Menschen über Vergangenheit, Gegenwart und Zukunft und die gesellschaftlichen Verhältnisse, die diese verbinden, verändern. So haben allgemeine Theorien der menschlichen Geschichte, wie der historische Materialismus und viele andere, für mich keine Berechtigung.

$D . R .:$ Wohl aber begrenzte Generalisierungen über soziale Konventionen einerseits, andererseits über die Konsequenzen von Handlungen ...

A.G.: . . die beiden Typen sozialwissenschaftlicher Generalisierung, die ich erwähnt habe. Auf der anderen Seite kann man die Bedeutung von Generalisierungen in den Sozialwissenschaften leicht überschätzen. Eine mindestens ebenso wichtige Art von Wissen, die Sozialwissenschaftler bereitstellen sollten, ist die Formierung neuer Konzepte oder Sichtweisen. Sie eröffnen alternative Handlungsmöglichkeiten in der Gesellschaft, denn jene, die z.B. gelernt haben, die Bildung der sozialen Welt auf andere Weise zu sehen, verändern die Gesellschaft auch in anderer Weise. Die sozialen Bewegungen der letzten Zeit zeigen das deutlich. Konzepte wie «Patriarchat» und eine allgemeine Aufmerksamkeit für Gender-Fragen ${ }^{18}$ sind hauptsächlich durch den Feminismus eingeführt worden und veranlassen nicht nur die unmittelbar gesellschaftlich Handelnden, sondern auch die "Gesellschaft» thematisierenden Soziologen und Geographen zu einer anderen Sicht und zu anderem Vorgehen.

D.R.: Sehen Sie bei dieser zweiten Art von Wissen, der Eröffnung neuer Sichtweisen, eine Verbindung zwischen der traditionellen Aufgabe der Kunst und jener einer kritischen Sozialwissenschaft?

A.G.: Ich würde Kunst nicht en bloc durch die Intention charakterisieren, Menschen neue Sichtweisen zu eröffnen. Immerhin war Kunst bis zur Moderne hauptsächlich darstellend, und sie mag auch tiefere Intentionen haben als diese. Ähnlichkeiten gibt es insofern, als sowohl die moderne Kunst wie auch die Sozialwissenschaften mit dem Projekt der Moderne verbunden sind, mit der Idee der dauernden Revision der Lebensform auf der Basis erwei- 
terten Wissens. Beide erforschen Möglichkeiten der Betrachtung der Welt, die noch nicht wahrgenommen worden sind. Aber das gilt nur für bestimmte Bereiche, auch der modernen Kunst.

D.R.: Ich möchte noch einmal auf den Feminismus zurückkommen: Welche Bedeutung hat die feministische Gesellschaftstheorie für die gegenwärtigen Sozialwissenschaften?

A.G.: Grundlegende Bedeutung - aus drei Gründen: Den ersten habe ich schon erwähnt, sie konfrontiert die etablierten Betrachtungsweisen einer, im wesentlichen von männlichem Handeln dominierten Sozialwissenschaft mit neuen, für diese Wissenschaft grundlegenden Konzepten. Zweitens wurden durch feministische Gesellschaftstheorien, und die Frauenbewegung allgemein, neue Themen auf die Forschungsagenda gesetzt. Eine Geschichte der Rolle der Frauen, oder die Beschreibung ihrer gegenwärtigen Lebensumstände, liefert nicht nur empirische, sondern auch theoretische Ergebnisse. Ein dritter Grund für die große Bedeutung feministischer Gesellschaftstheorie liegt in deren Herausforderung an bestehende Gesellschaftstheorien, in ihre theoretischen Erklärungen eine Gender-Dimension einzubeziehen. Meine Arbeiten haben diese Dimension bisher nicht wirklich beachtet, aber ich beschäftige mich jetzt damit. Eine Frage z.B. ist, ob ich von sozial Handelnden sprechen kann, ohne sie als Handelnde einer Gender-Position zu spezifizieren, ... aber ich habe hier noch keine definitiven Antworten.

D.R.: Ich möchte noch ein anderes Thema anschneiden: Bei der Beschreibung Ihrer Strukturierungstheorie sprechen Sie von der «dialektischen Relation» zwischen Handlung und Struktur, von sozialen Systemen als «strukturierte Totalitäten», oder von «Momenten", in denen die soziale Struktur aktualisiert wird. Kann man sagen, die Strukturierungstheorie beinhalte eine dialektische Auffassung von «Gesellschaft»?

A.G.: Nein, wenn man den Begriff der «Dialektik» präzise verwendet und ihn mit einem philosophischen Gedankensystem, wie jenem von Hegel oder zu einem gewissen Grade auch von Marx (obwohl er den Begriff der «Dialektik», soweit ich weiß, nicht konsistent gebraucht hat) in Verbindung bringt. Ja, wenn man ihn eher locker gebraucht. Ich glaube, da $\beta$ Widersprüche zu einem gewissen Grad die treibende Kraft sozialer Veränderungen bilden, aber sicher nicht die einzige. Und es gibt so etwas wie die Verschmelzung von Gegenteilen ... Ich verwende den Begriff der Dialektik manchmal, aber nicht besonders gerne. Bei Marx hat man ab und zu den Eindruck, er verwende ihn, um zu verdecken, was in Wirklichkeit eine genauere Analyse verlangen würde.
$D . R .:$ Und eine Verwendung des Begriffes «Dialektik» im präzisen Sinne? Ich verstehe nicht, wie die Strukturierungstheorie nicht auf dialektischer Logik beruhen sollte, wie sie dann aber ohne einen Begriff von Totalität und ohne Anerkennung eines übergeordneten Vernunftprinzipes - wenigstens implizit-auskommen könnte-als Theorie, meine ich. Könnten Sie Dialektik in ihrer Theorie rein als Logik der Veränderung sehen, ohne Verbindung mit einer bestimmten (z. B. Hegelschen) Interpretation der abendländischen Geschichte oder einer (z. B. Marxschen) Erklärung der Entwicklung des Kapitalismus?

A.G.: Auch das nicht, denke ich, wenn es bedeutet, daß eine Totalität in gewissem Sinn mit den Teilen eines Systems verbunden ist... Ich glaube nicht, $\mathrm{da} B$ ein soziales System sich so verhält. Es ist einfacher, und zugleich komplizierter, als das. Sicher verwendet man für eine bestimmte Handlung ein Wissen über gesellschaftliche Konventionen und Regeln, und die hat man weder selbst erfunden, noch gelten sie allein subjektiv. Sie verbinden die Akteure und regeln ihre Beziehungen in systematischer Weise, aber sie sind kein Bestandteil einer vagen Totalität. Ich weiß nicht genau, was damit gemeint sein soll ... daß Teile des Gesellschaftlichen vom Handelnden internalisiert wurden, in der Art, wie Durkheim meinte... aber das scheint keine brauchbare Idee zu sein. Ich verwende den Begriff der Totalität nicht oft, weil es so etwas wie die Totalität nicht gibt. Es gibt verschiedene Ebenen der Organisation sozialer Systeme in Raum und Zeit, und «Gesellschaft» mit «Totalität» zu verbinden würde einer einzelnen Systemebene falsche Priorität geben. Wie ich Gesellschaft nicht als Gegenüberstellung von Individuum und Sozialem beschreiben würde, so möchte ich auch die Begriffe «Totalität» und «Teile» nicht verwenden. Nicht, daß ich da keine Beziehung sehe, aber ich spreche lieber von Handelnden und Struktur und System, und betrachte diese als in ziemlich komplexer Weise miteinander verbunden.

D.R.: Eine konkretere Frage betrifft die Themen Gewalt, Herrschaft und Kontrolle. Im Buch «Nation State and Violence» haben Sie diese Themen neuerlich aufgegriffen und dabei nicht nur das sozialwissenschaftliche Konzept von «Macht» differenziert, sondern auch versucht, die dualistische Trennung zwischen voluntaristischen und strukturellen Machtbegriffen aufzuheben.

Geographen, die sich auf Ihre Arbeiten berufen haben, ergriffen bisher hauptsächlich die Kontextualität Ihrer Strukturierungstheorie auf, weniger aber die darin vorgezeichnete Möglichkeit der Analyse räumlicher Dimension von Macht. Sehen Sie insbesondere auf diesem Gebiet eine Chance zu- 
gewinnbringender Zusammenarbeit mit Geographen, insbesondere aus dem Bereich der Politischen Geographie?

A.G.: Zunächst einmal sind Soziologen und Geographen in bezug auf ein Interesse an «Macht» auf sehr allgemeiner Ebene über den Begriff der «räumlich-zeitlichen Ausdehnung (time-space distanciation)» verbunden. Ich habe in verschiedenen Büchern dargelegt, welche zentrale Rolle die Art und Weise, in der verschiedene Gesellschaften Zeit und Raum fassen, für ihre Ordnung spielt - was andererseits wieder direkt ihre Machtsysteme impliziert. Ich habe das z.B. an der Bedeutung der Schrift für die Entwicklung traditioneller Gesellschaften zu zeigen versucht. Die Schrift ist ein Mittel zur - oder führt zur - Reorganisation des sozialen Zusammenlebens über Zeit und Raum, aber sie ist auch ein Mittel zur Entwicklung von Macht und gestattet $\mathrm{Ge}$ sellschaftsformen, die über Ackerbauen, Jagen und Sammeln hinausgehen. Es gibt aber auch noch eine spezifischere Verbindung zwischen Soziologen und
Geographen. Sie betrifft die Territorialität von Staaten. In «Nation, State and Violence» habe ich argumentiert, daß besonders Soziologen die Territorialität nicht im Zusammenhang - in den vollen Konsequenzen ihres Zusammenhanges - mit Staaten betrachtet haben. Ich habe z.B. beschrieben, wie die Territorialität traditioneller Staaten sich grundsätzlich von jener der Nationalstaaten unterscheidet. Das hängt mit der Art zusammen, in der ein Territorium in Machtsysteme einbezogen wird. Hier gibt es noch eine Menge lohnende Arbeit. Eine dritte Verbindung könnte im Interesse für den Einfluß militärischer Macht, militärischer Gewalt auf die gesellschaftliche Entwicklung liegen. Diese Frage ist von Sozialwissenschaftlern zwar aufgegriffen worden, aber Geographen und Soziologen könnten hier sicherlich fruchtbar zusammenarbeiten, denn Kriegführung und militärische Macht beziehen auch geopolitische Erwägungen mit ein. Dies ist eine Fragestellung einer kritischen Sozialwissenschaft, die ich besonders in der heutigen Zeit für sehr wichtig halte.

\section{Anmerkungen:}

1 Ausgangspunkt für diesen Beitrag war ein Interview im März 1988 in Cambridge. Nachdem ich es gekürzt und übersetzt hatte, wurde die endgültige Fassung von A. Giddens überprüft. Ich möchte mich an dieser Stelle für seine Geduld bedanken. Dank auch an Min. S. S. für die Gespräche und an $\mathrm{E}$. Ye für das Management.

2 Eine kurze Zusammenfassung der Strukturierungstheorie gibt Giddens selbst im Buch: "A Contemporary Critique of Historical Materialism" (26-29), und im ersten Kapitel von "The Constitution of Society". Dort finden sich auch Anwendungsbeispiele. Prägnante Zusammenfassungen von Geographen sind z.B. Pred (1983, Storper (1985), Thrift (1983, 1985) und Gregory (in Vorbereitung, a. und b.).

3 D. Gregory (1984).

4 Times Literary Supplement

5 z. B. D. Gregory (1982), A. Pred (1986), Dear and Moss (1986).

6 z.B. in den britischen CURS- und Sussex-Forschungsprogrammen.

7 Als Beispiel für einige wenige Ausnahmen sei die Arbeit von E. Binder (1988) genannt.

8 Siehe meine Frage zur Dialektik im folgenden Interview.

9 Mit raum-zeitlicher Materialität erfaßt Giddens eine Qualität existentieller Gegebenheit, raum-zeitlichen Kontext bezeichnet er als "locale", raum-zeitliche Distanz als "space-time distanciation" (d.h. Prozeß räumlich-zeitlicher Ausdehnung), die Begrenzung von Räumen (territorielle Verfügungsbeschränkung) heißt in seiner Terminologie "regionalization".

10 Dieser Vortrag mit dem Titel: «The role of space in the con- stitution of society" ist in: Steiner, D. Jaeger, C. und Walther, P., (in Vorbereitung) veröffentlicht.

11 Mit "Gesellschaft als rekursiver Prozeß" ist die laufende Wechselwirkung ("Strukturierung") zwischen Handlungen in konkreten Umständen und einer ideellen Ordnung von Regeln und Verfügungsgewalten gemeint.

12 Siehe z.B. die Zusammenfassung von K. Aerni (1986).

13 Siehe auch R. Naegelis Zusammenfassung der dortigen Diskussion (Naegeli, in Vorbereitung).

14 Im Buch "Central Problems in Social Theory" (1977, p. 165-97) hat sich Giddens mit dem Ideologiebegriff befaßt. Er lehnt darin die traditionelle Bedeutung von "ldeologie" als Gegenstück zu "Wissenschaft" ab und betrachtet sie eher als mögliche Dimension jeder Form des Diskurses, als dessen Herrschaftsdimension. Durch die Verdeckung und Verdrängung von Widersprüchen sowie durch Verdinglichung werden in ihr partikuläre Interessen gegen Gesamtinteressen durchgesetzt.

15 K. Mannheim (1929): Ideologie und Utopie.

16 Siehe J. Habermas, 1976, 1982.

17 Beispielsweise in der Diskussion des Aufsatzes von C. Jäger (1987) am Schweizer Geographentag in Fribourg (siehe Naegeli, 1987), oder in der englischen Debatte "Reconsidering social theory" zwischen D. Harvey und P. Saunders, P. Williams, und einer Reihe anderer Geographen (Society and Space, Vol. 5/87, p. 367-434), oder - mit expliziertem Bezug auf Giddens - in D. Harvey and A. Scott (in Vorbereitung) und D. Gregory (i. V.).

18 Ich verwende hier und in der Folge den englischen Begriff "gender", um die im Deutschen nicht gebräuchliche Unterscheidung zwischen biologischen Kategorien ("sex") und sozialisationsbedingten Kategorien ("gender») zu bewahren. 


\section{Literatur:}

AERNI, K. (1986): Die Rolle der Geographie in der Gesellschaft. (Einleitung zu den Referaten zum Schweizer Geographentag 1986). In: Geographia Helvetica, 3, 126-127.

BINDER, E. (i. V.): Auf der Spur des Neuen. Notizen zur Theorie der Strukturierung von A. Giddens. In: AUFHAUSER, E. und GIFFINGER, R. (Hrsg.): Perspektiven Regionalwissenschaftlicher Forschung. Mitteilungen der Arbeitsgemeinschaft für Methoden der Regionalforschung, Band 18, Wien.

BOESCH, M. (1986): Schweizer Geographie am Wendepunkt. Überlegungen zu einer normativen Metatheorie. In: Geographia Helvetica 3/86, 147-154.

DEAR, M. and MOSS, A. (1986): Structuration theory in urban analysis. In: Environment and Planning $A, 18, p$. 213-252.

GIDDENS, A. (1977): Central Problems in Social Theory. Hutchinson, London.

GIDDENS, A. (1977): Studies in Social and Political Theory. Hutchinson, London.

GIDDENS, A. (1981): A Contemporary Critique of Historical Materialism. Vol. 1: Power, Property and the State. Macmillan, London.

GIDDENS, A. (1981): The Class Structure of Advanced Societies. Hutchinson, London.

GIDDENS, A. (1982): Profiles and Critiques in Social Theory. Macmillan, London.

GIDDENS, A. (1984): The Constitution of Society. Polity, Cambridge.

GIDDENS, A. (1984): Interpretative Soziologie. Campus, Frankfurt. (Engl.: New Rules of Sociological Method, 1976).

GIDDENS, A. (1985): The Nation State and Violence (Vol. 2 of: A Contemporary Critique of Historical Materialism). Polity Press, Cambridge.

GIDDENS, A. (1987): Social theory and modern sociology. Polity, Cambridge.

GIDDENS, A. (in Vorbereitung): The role of space in the constitution of society. Vortrag an der ETH Zürich. In: STEINER, D., JAEGER, C. und WALTER, P.

GREGORY, D. (1982): Regional Transformation and Industrial Revolution. Macmillan, London.

GREGORY, D. (1984): Space, time and politics in special theory: an interview with Anthony Giddens. In: Society and Space, 2, 123-132.

GREGORY, D. (1987): Areal Differentiation and Post-Modern Geography. In: Walford, R. (ed.): New Horizon in Geography. Hutchinson, London.

GREGORY, D. (i. V., a): Presences and absences: timespace relations and structuration theory. In: HELD, D. and
THOMSON, J.: Critical Theory of the Industrial Societies. Cambridge Univ. Press, Cambridge.

GREGORY, D. (i. V., b): The geographical imagination: social theory and human geography. Hutchinson, London.

GREGORY, D. (1986): On duality and dualism. The case of structuration and time geography. In: Progress in Human Geography. 2, 184-205.

HABERMAS, J. (1976): Was heißt Universalpragmatik? In: Apel, K. O. (Hrsg.): Sprachpragmatik und Philosophie, 174-272, Suhrkamp, Frankfurt.

HABERMAS; J. (1982): Vorbereitende Bemerkungen zu einer Theorie der kommunikativen Kompetenz. In: Habermas, J. und Luhmann, N.: Theorie der Gesellschaft oder Sozialtechnologie - Was leistet die Systemforschung? 2. Aufl. 101-141, Suhrkamp, Frankfurt.

HARVEY, D. and SCOTT, A. (i. V.): The practice of human geography. Theory and empirical specificity in the transition from Fordism to Flexible Accumulation. In: W. Macmillan (Ed.): Remodelling Geography. Basil Blackwell, Oxford.

JAEGER, C. (1987): Theorie und integrative Ansätze in der Geographie. Bericht der Arbeitsgruppe «Theorie und integrative Ansätze" zum Schweizer Geographentag in Fribourg 1987. Reproduziert in: STEINER, JAEGER und WALTHER 3-14.

NAEGELI-OERTLE, R. (i. V.): Nachlese zur Theoriediskussion am Geographentag in Fribourg, 1987. In: STEINER, D., JAEGER, C. und WALTHER, P.

PRED, A. (1983): Structuration and place: on the becoming a sense of place and structure of feeling. In: Journal of the Thoery of Social Behaviour, Vol. 13, 157-186.

PRED, A. (1986): Place, Practice and Structure. Polity, Cambridge.

REICHERT, D. (1988): Der Pfad des Sisyphos. Zur Zirkularität in den Grundlagen humangeographischer Forschung. Dissertation am Geographischen Institut der Universität Wien.

STEINER, D., JAEGER, C. und WALTHER, P. (i. V.): Jenseits der mechanistischen Kosmologie. - Neue Horizonte für die Geographie. Berichte und Skripten Nr. 36, Geographisches Institut der ETH Zürich.

STORPER, M. (1985): The spatial and temporal constitution of social action: A critical reading of Giddens. In: Society and Space, 3, 407-424.

THRIFT, N. (1983): On the determination of social action in space and time. In: Society and Space, Vol. 1 (1983), 23-57.

THRIFT, N. (1985): Bear and Mouse or bear and tree? Anthony Gidden's reconstitution of social theory. In: Sociology, Vol. 19, 609-623.

WERLEN, B. (1987): Gesellschaft, Handlung, Raum: Grundlagen handlungstheoretischer Sozialgeographie. Steiner, Stuttgart. 